\section{Systemic MMP inhibition for periodontal wound repair: results of a multi-centre randomized- controlled clinical trial}

Gapski R, Hasturk H, Van Dyke TE, Oringer RJ, Wang S, Braun TM, Giannobile WV. Systemic MMP inhibition for periodontal wound repair: results of a multi-centre randomized-controlled clinical trial. J Clin Periodontol 2009; 36: 149-156. doi: 10.1111/j.1600-051X.2008.01351.x.

\begin{abstract}
Aim: This multi-centre, prospective, controlled trial was designed to examine the biological response of the matrix metalloproteinase(MMP) inhibitor subantimicrobial dose doxycycline (SDD) combined with access flap surgery on periodontal wound repair in patients with chronic severe periodontitis.

Material and Methods: Seventy subjects were enrolled into a 12-month, randomized, placebo-controlled, double-masked trial to evaluate disease response to 6 months therapy and "wash-out" of either placebo+surgery or SDD (20 mg b.i.d.)+ surgery. Primary outcome measure included clinical attachment levels (CAL) and secondary outcomes included probing depth (PD), bleeding on probing (BOP), as well as gingival crevicular fluid bone marker assessment [collagen telopeptides

(ICTP)]. These measurements were taken at baseline through 12 months post-surgery and drug administration.

Results: Patients treated with SDD and surgery demonstrated stronger reductions in PD in surgically-treated sites of $\geqslant 7 \mathrm{~mm}$ as well as gains in CAL $(p<0.004)$.

Furthermore, SDD+surgery resulted in short-term reductions in ICTP levels compared with placebo. Rebounds in ICTP levels and clinical parameters occurred when SDD was withdrawn.

Conclusions: The results from this multi-centre study suggests that SDD in combination with surgery improves the short-term response of periodontal therapy by reducing $\mathrm{PD}$, increasing $\mathrm{CAL}$ gain and inhibiting early stage bone resorption.
\end{abstract}

\author{
Ricardo Gapski ${ }^{1}$, Hatice Hasturk ${ }^{2}$, \\ Thomas E. Van Dyke ${ }^{2}$, Richard J. \\ Oringer $^{3}$, Shufang Wang ${ }^{4}$, Thomas $M$. \\ Braun $^{1,4}$ and William V. Giannobile ${ }^{1}$ \\ ${ }^{1}$ Michigan Center for Oral Health Research \\ and Department of Periodontics and Oral \\ Medicine, School of Dentistry, University of \\ Michigan, Ann Arbor, MI, USA; ${ }^{2}$ Department \\ of Periodontology and Oral Biology, Boston \\ University, Boston, MA, USA; ${ }^{3}$ Department \\ of Periodontics, Stony Brook University, \\ Stony Brook, NY, USA; ${ }^{4}$ Department of \\ Biostatistics, School of Public Health, \\ University of Michigan, Ann Arbor, MI, USA
}

Key words: doxycycline; matrix metalloproteinases; MMP; periodontal; wound healing

Accepted for publication 19 October 2008
It is generally accepted that, although periodontal disease is initiated by the subgingival microflora, inflammatory mediators released by host cells within the gingival tissues are major factors responsible for the destruction of the supporting periodontal tissues (Kornman

\section{Conflict of interest and source of} funding statement

This study was supported by CollaGenex Pharmaceuticals, Inc., NIH/NCRR UL1RR024986, and the Delta Dental Fund.
2008). Microbial virulence factors trigger the release of host-derived enzymes such as proteases (e.g., matrix metalloproteinases or MMPs) that can lead to destruction of tooth-supporting tissues (Slots \& Genco 1984, Socransky \& Haffajee 1991, Gopalsami et al. 1993). MMPs are a family of endopeptidases with activity against most extracellular macromolecules (Pussinen et al. 2007). Collagenases, a subclass of the MMP family, are a group of enzymes capable of disrupting the triple helix of type I collagen in physiological conditions, the primary structural component of the periodontium. A number of physiological events [i.e., embryonic development and tissue remodeling (Shi et al. 2007)] and pathologic conditions such as arthritis (Murphy \& Nagase 2008) and cancer (Lopez-Otin \& Matrisian 2007) are characterized by heightened MMP activity. Studies have shown elevated levels of collagenases and other hostderived proteinases (e.g., cathepsins, elastase, tryptases/trypsin like proteinases) in inflamed gingiva, gingival crevicular fluid (GCF), and saliva of 
humans with periodontal disease (Golub et al. 1976, 1990, Sorsa et al. 1988). Therefore, the determination that periodontal tissue destruction is primarily due to the host response has created areas of research directed at altering an individual's reaction to the bacterial challenge. Hence, new methods of host modulatory therapy for periodontal disease are being focused beyond the traditional control of tooth-associated microbial biofilms.

Tetracyclines (TCNs; including the semi-synthetic analogues, minocycline and doxycycline) are capable of inhibiting mammalian collagenase activity (Golub et al. 1997, 2001). TCN's inhibitory effect on collagenases appears to occur through the drug's ability to bind to zinc cations as well as calcium ions in the catalytic domain of the molecule (Golub et al. 1983, 1987). Furthermore, administration of low doses of subantimicrobial dose doxycycline (SDD; a tetracycline analogue) given $20 \mathrm{mg}$ b.i.d. rather than the 50 $100 \mathrm{mg}$ capsules, typically administered as commercially available antibiotics, effectively inhibit collagenase activity in the gingival tissues as well as in GCF (Golub et al. 1998) without exerting direct effects on the subgingival microorganisms. Several clinical studies have demonstrated improvements in gingival healing when SDD is used in combination with traditional dental prophylaxis or SRP (Caton et al. 2000, 2001, Novak 2002, Preshaw et al. 2004). More recently, a modified-released SDD formulation containing $40 \mathrm{mg}$ doxycycline once a day demonstrated similar positive results (Preshaw et al. 2008).

Many of the crucial cellular responses of early post-surgical wound healing, such as inflammatory infiltration, angiogenesis and re-epithelialization, are made possible through the action of MMPs (Tarlton et al. 1997). Expression of MMPs such as collagenases is elevated in acute wounds, and still greater levels are found in chronic wounds, indicating that uncontrolled proteolysis is a characteristic of retarded healing (Tarlton et al. 1997, Neely et al. 2000, Gu et al. 2002). Several members of the MMP family function as positive and, at times, negative regulators of repair processes (Gill \& Parks 2008). MMPs have demonstrated functions in bone including degradation of collagen and other components of the bone matrix, migration and survival of bone cells, endochondral bone formation and bone resorption (Varghese 2006). Abnormal expression of MMPs may also lead to pathological conditions affecting bone and cartilage (Giannobile 2008). Our group previously demonstrated in a single centre, proof-of-concept investigation that the utilization of a 6-month dosing of SDD in combination with access flap surgery could reduce GCF levels of collagen telopeptides (ICTP) and reduce pocket depth in patients with severe periodontitis (Gapski et al. 2004, Goodson 2004). However, given the limited sample, a more expanded, multi-centre investigation was warranted to better characterize the responses to therapy. Therefore, the aim of this multi-centre study was to evaluate the effect of a MMP-inhibitor (MMPi) in the post-surgical would healing in a larger population with chronic periodontitis.

\section{Materials and Methods Study population}

A written informed consent was provided for individuals willing to participate in this trial that met the inclusion and exclusion criteria outlined in a protocol approved by Institutional Review Board of Academic Health Centres at the University of Michigan, Boston University and the Stony Brook University. Three calibrated surgeons and three separate individual clinical examiners were utilized from each Clinical Centre. In order to minimize the impact of inter-examiner variability, all clinical measurements were conducted by the same examiner from each center for the duration of the study. Before study commencement, the study examiner was trained and practiced in each of the clinical measurements. Duplicate measurements were compared to ensure a standard deviation of $<1.0 \mathrm{~mm}$ for repeated within-subject probing depth (PD) and clinical attachment level (CAL) measures. The human clinical trial was performed in accordance with CONSORT guidelines (Moher et al. 2001). Seventy subjects (aged 30-75 years) diagnosed with chronic severe periodontitis were enrolled into a double-masked, randomized, placebo-controlled, prospective, parallel arm multi-center trial during the period of 1999-2003. Patients were recruited from November of the year 1999 to July of 2002. These patients were followed until July of 2003 (Fig. 1). Pertinent inclusion criteria included patients with at least three teeth in the same sextant demonstrating both PD and $\mathrm{CAL} \geqslant 5$ to $\leqslant 12 \mathrm{~mm}$ and bleeding on probing (BOP) with at least 10 teeth in the functional dentition. Exclusion criteria included patients chronically treated (i.e., 2 weeks or more) with any medication known to affect periodontal status (i.e., antibiotics or NSAIDS); those who received antibiotics within 90 days of baseline (BL); patients possessing clinically significant or unstable organic diseases; patients displaying compromised healing potential such as those with con-

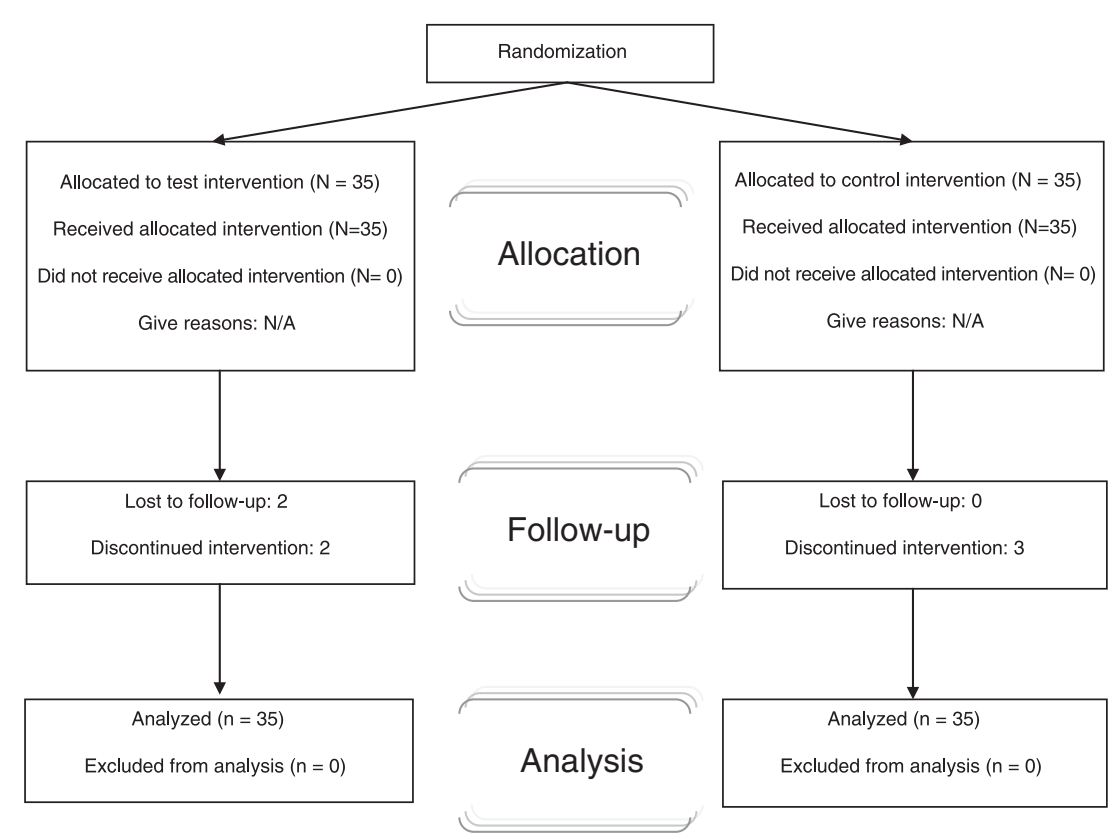

Fig. 1. Patient flow through study. 
nective tissue disorders or bone metabolic diseases; patients with conditions necessitating antibiotic prophylaxis; females who were pregnant (as determined by positive urine pregnancy test at the pre-screening visit) or lactating, or female patients who were of childbearing potential and not utilizing birth control or abstinence; patients with documented allergies to TCNs; heavy cigarette smokers $(\geqslant 2$ packs/day); active infectious diseases, immunocompromised or taking steroid medications.

\section{Study design}

Patients eligible to participate in the study received full mouth scaling and root planing within 90 days before BL. Fourteen days before surgery, subjects were re-examined (screening visit) and inclusion/exclusion criteria were reviewed. Subjects who still qualified for the study and required access flap surgery in a minimum of one sextant were randomized to receive either doxycycline hyclate $20 \mathrm{mg}$ b.i.d. (Periostat ${ }^{\circledR}$, Galderma Labs, Fort Worth, TX, USA) or placebo capsules b.i.d. for the treatment period of 6 months. Full mouth manual clinical measurements were recorded for all teeth within 2 weeks of the BL appointment. Six sites were measured around each tooth in the mouth (mesiobuccal, buccal, distobuccal, distolingual, lingual and mesiolingual) for PD, CAL and BOP. At the same visit, GCF samples were collected on teeth assigned to receive surgery. Examiners were trained to minimize intra-rater (i.e., intra-examiner) variability with respect to the surgical operation and clinical measures performed.

Surgical procedures were then performed at BL within 14 days from the screening visit. Patients returned for follow-up examinations at months 1, 2, 3, 6, 9 and 12 following BL. Full mouth manual probing measurements and GCF ICTP levels were assessed at BL and months 2, 3, 6, 9 and 12. The subjects received periodontal maintenance therapy at 3, 6, 9 and 12 months following randomization and periodontal surgery. The study design is shown in Table 1.

\section{Surgical procedures}

Briefly, following local anesthesia, treatment sextants (sites having $\geqslant 5 \mathrm{~mm}$ pocket depth) received access flap surgery, which consisted of submarginal primary incisions and full-

Table 1. Clinical trial longitudinal measures

\begin{tabular}{lccccccccc}
\hline Eventltime points & P-SC & SC & BL & M-1 & M-2 & M-3 & M-6 & M-9 & M-12 \\
\hline Medical history & X & & & & & & & & \\
Informed consent & X & & & & & & & & \\
SRP & X & & & & & & & & \\
Full mouth measurements & X & X & X & & & X & X & X & X \\
Urine pregnancy test & & X & & & & & X & & \\
Surgery & & & X & & & & & \\
C-telopeptide level & & & X & X & X & X & X & X \\
Maintenance therapy & & & X & & X & X & X & X \\
Dispense medication & & & & & & X & & & \\
Drug accountability & & & & & & X & X & & \\
\hline
\end{tabular}

P-SC, pre-screening; SC, screening; M, month; SRP, scaling and root planing; $\mathrm{X}=$ timing of procedue

thickness mucoperiosteal flap reflection. The tooth root surfaces were debrided, scaled and root planed using hand and sonic instrumentation. Osseous recontouring (osteoplasty) when indicated was performed for flap adaptation. The tissues were repositioned with Polyglactin 910 (Vicryl, Ethicon Inc., Somerville, NJ, USA) or silk suture materials. After surgery, patients received pain control medication (an acetaminophencontaining analgesic) when needed and chemical plaque control $(0.12 \%$ chlorhexidine rinse) for 14 days.

\section{Bone marker (ICTP) analysis}

GCF was collected before clinical measurements. Gingival tissue surrounding the site to be sampled was dried with gauze, and supragingival plaque was removed. A sterile methylcellulose strip (Periopaper ${ }^{\circledR}$ : IDE-Interstate, Amityville, NY, USA) was gently placed into the mesiobuccal aspect of each tooth sulcus or pocket until slight resistance was felt, and the GCF collected for $10 \mathrm{~s}$. Following collection, the sample was kept on ice for transport to the laboratory and stored at $-80^{\circ} \mathrm{C}$. The frozen samples were then thawed at room temperature and proteins were eluted through five centrifugations at $1500 \times \mathrm{g}$ for $5 \mathrm{~min}$ with $20 \mu \mathrm{l}$ phosphate-buffered saline ( $\mathrm{pH}$ 7.4) containing $15 \mathrm{nM}$ aprotinin, $1 \mathrm{mM}$ PMSF and $0.1 \%$ of human serum albumin as previously described (Giannobile et al. 1995). GCF ICTP levels were quantified using radioimmunoassay (RIA; DiaSorin Inc., Stillwater, MN, USA).

\section{Dispensing of study medication/ assessment of compliance}

At the screening visit, qualifying patients were assigned a code and were then randomized by a computer software program to the drug or placebo group using block randomization method. At the BL visit, each patient was issued a 3-month supply of coded study medication including one container for $\mathrm{AM}$ and another for PM intake. Three months following surgery, subjects returned residual capsules and received an additional 3-month supply. The residual capsules were counted and retained at 3 and 6 months after BL. Patient compliance (as a percentage) with the dosing schedule was recorded.

\section{Adverse events}

All adverse events were recorded. The need to capture this information was not dependent upon whether the adverse event was associated with the use of the study medication or not. Adverse events resulting from concurrent illnesses or reactions to concurrent medications were also recorded. Each adverse event was evaluated for duration, intensity and relationship with the study medication or other causes. When the different intensities occurred more frequently than once a day, the maximum intensity for the event was recorded. If the intensity category changed over a number of days, then these mini-events or changes were recorded separately (i.e., having distinct onset days). The investigator used the criteria below as guidelines for determining the relationship of the adverse event to the study drug: (i) a temporal relationship existed between the event and the use of the drug; (ii) re-administration of study medication resulted in reappearance or worsening of the reaction; (iii) previous experience with the suspected drug resulted in a similar reaction; and (iv) the event was not related to any concomitant disease, pre-existing condition, other drug therapy or environmental 
factors. One of the following determinations will then document the relationship of the adverse event to the study drug: not related, possible and probable.

\section{Statistical analyses}

Based upon data presented in Fig. 1 of Golub et al. 1997, we have $80 \%$ power for detecting a $30 \mathrm{pg} /$ site difference in mean ICTP, assuming a standard error of about $50 \mathrm{pg} / \mathrm{site}$ and type I error rate of $5 \%$, between 35 control patients and 35 SDD patients. For analysis, the primary outcome measure was considered CAL and secondary outcomes included PD, BOP, as well as GCF bone marker assessment ICTP. Patient group differences in demographics were compared with a Wilcoxon's rank sum test for continuous measures and a $\chi^{2}$ test of association for categorical measures. For each time point, site-specific levels of CAL, PD and BOP were first averaged across each tooth of each subject. The subject-level means of CAL, PD, BOP and ICTP were estimated via generalized estimating equations (GEE) with an independence working correlation matrix; statistical significance was based upon GEE robust variance estimates to account for: (1) correlation of teeth from the same subject, and (2) temporal correlation of values from the same tooth. Results presented are based upon GEE models with effects for treatment, time and their interaction. Adjustment for differences among sites was also examined, but later excluded due to lack of statistical significance. Standard errors presented for each outcome at each time point are based upon the robust GEE variance estimates. Statistical significance was defined as any $p$ value $<0.05$; No adjustment was made for comparisons among multiple time points. Subjects with missing data were included in all analyses to the extent of the actual data that was collected on those subjects, i.e., an intent-to-treat analysis was used, and no missing data were inputted.

\section{Results}

Demographics - BL data

A total of 70 patients entered into the study; 35 were randomized to the SDD group and 35 to the placebo group. Thirty-two out of 35 patients in the drug group and 33 out of 35 in the placebo group completed the study for

Table 2. Baseline mean demographics, clinical ICTP characteristics of the subject population

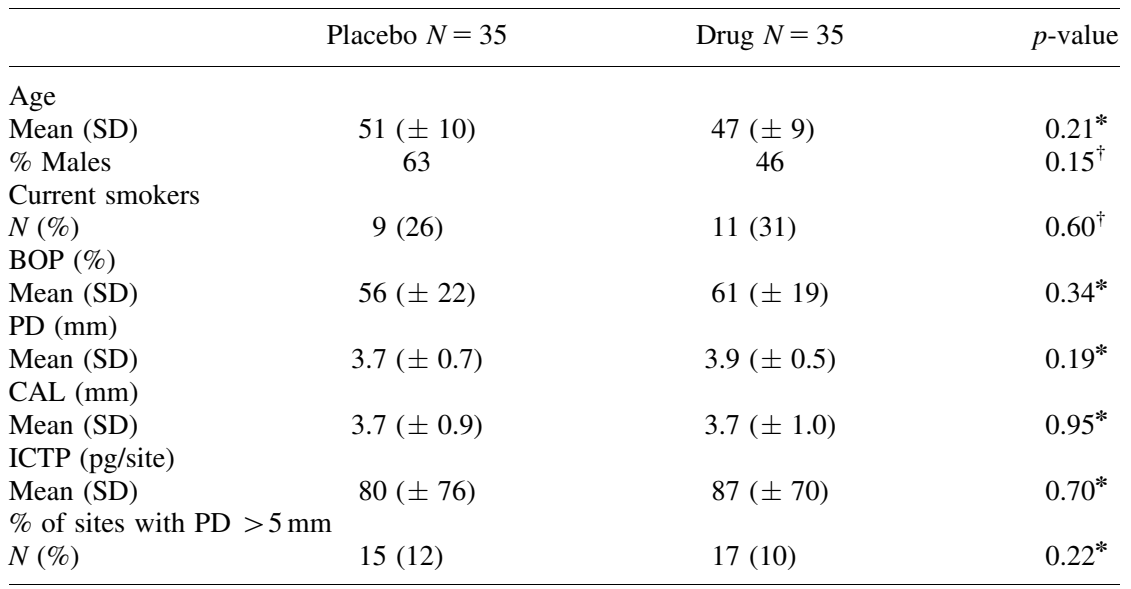

*By Wilcoxon's rank sum test.

${ }^{\dagger}$ By $\chi^{2}$ test of association.

$N$, number; SD, standard deviation, BOP, bleeding on probing; PD, probing depth; CAL, clinical attachment level; ICTP, collagen telopeptide.
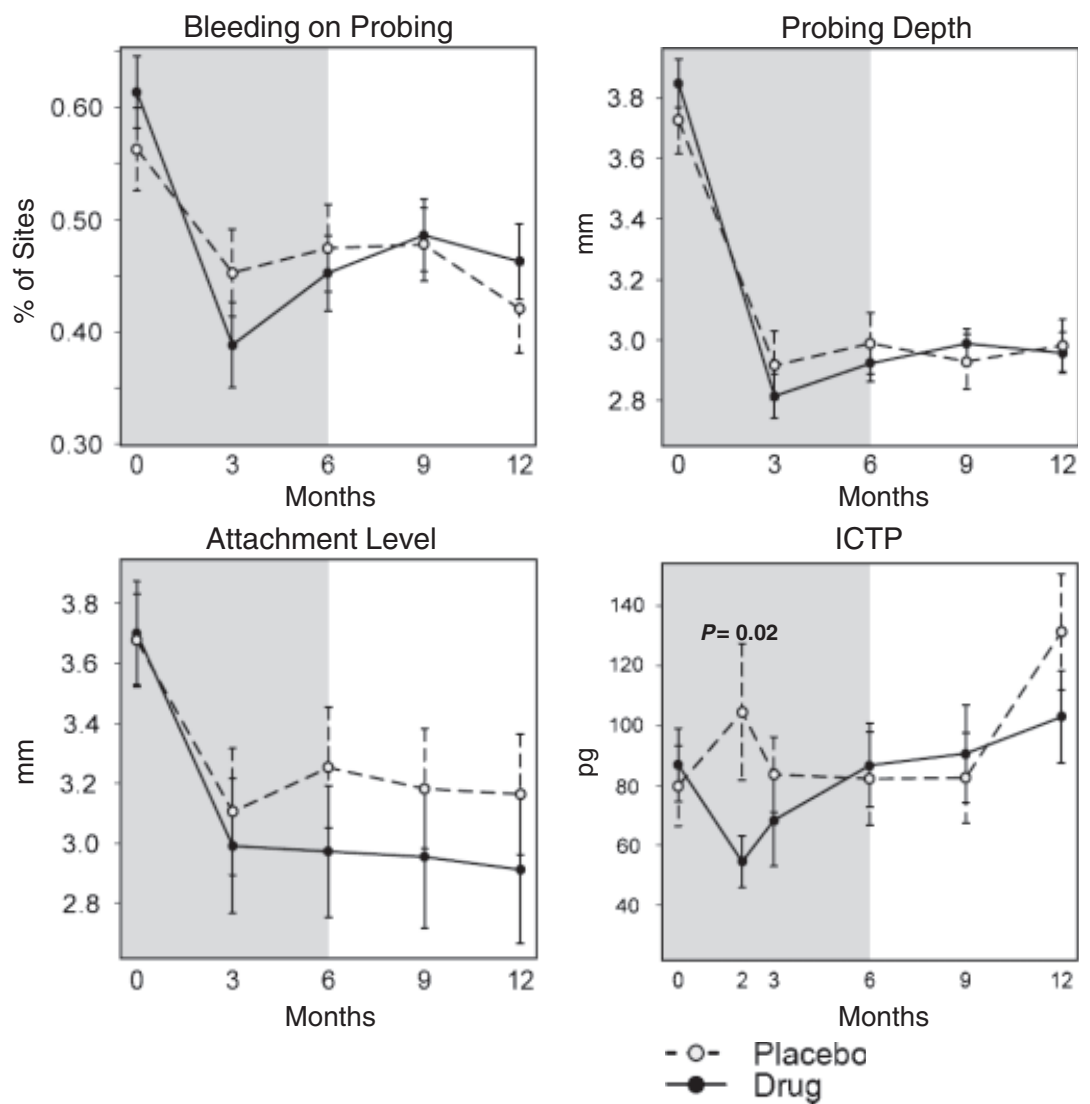

Fig. 2. Effect of subantimicrobial dose doxycycline (SDD) or placebo in combination with surgery on clinical parameters and collagen telopeptides (ICTP) for all sites receiving surgery. The shaded background depicts the time of SDD or placebo administration. The bars represent per-patient standard errors. $p$ values indicate significant treatment group differences at a time point as determined by generalized estimating equations (GEE). $N=70$ subjects.

an overall drop-out rate of 7.1\%. At BL, there were no statistically significant differences between groups regarding age and gender (Table 2). Twenty-six percent of SDD subjects reported cur- rent use of tobacco and $31 \%$ in the placebo group (Table 2, $p=0.6$ ). In addition, no significant differences were noted between the treatment groups with respect to BL clinical para- 
meters (PD, BOP and CAL), ICTP levels and percentage of sites with PD > $5 \mathrm{~mm}$ (Table 2).

\section{Compliance}

The mean overall drug compliance for the SDD-treated patients was $88.4 \%$ (range 0-114\%) while the placebo-treated individuals displayed $89.2 \%$ compliance (range 3-100\%). Patients had a tendency to be slightly more compliant in the morning than in the evening (90.8\% versus $86.7 \%$, respectively; $p=0.003)$. One patient in the drug group showed low compliance (18\%). Further, there were no statistical significant effects of compliance on any treatment differences.

Treatment outcomes - responses in clinical parameters and ICTP levels

\section{Pooled surgical sites (All PD strata)}

The mean per-patient means in BOP, PD, CAL and ICTP from BL for all surgical sites is shown in Fig. 2. As expected, both placebo and test groups demonstrated a statistically significant reduction in $\mathrm{PD}$ compared with $\mathrm{BL}$, however, no differences between groups was found. Surgical therapy resulted in mean CAL gains in both groups $(p<0.05)$. In addition, SDD-treated subjects demonstrated statistically significant reduced GCF ICTP levels compared with placebo immediately after the surgery ( 2 months, $p=0.03$ ).

\section{Moderate sites (BL PD 5-6 mm)}

Mean per-patient averages in BOP, PD, CAL and ICTP levels from BL at surgical sites with a BL PD of $5-6 \mathrm{~mm}$ is shown in Fig. 3. Consistent with results from pooled PD strata, there were significant reductions in $\mathrm{PD}$ and gains in CAL compared with BL for both drug and control groups. In addition, SDD group demonstrated a statistically significant decreased expression of GCF ICTP levels compared with placebo immediately after the surgery (2 months, $p=0.001)$. There was a statistically significant reduction in percentage of BOP sites at 3 months between drug and placebo groups $(p=0.02)$. Both SDD and placebo groups showed comparable levels in percentage of sites BOP after the patients discontinued drug therapy $(p>0.05)$.
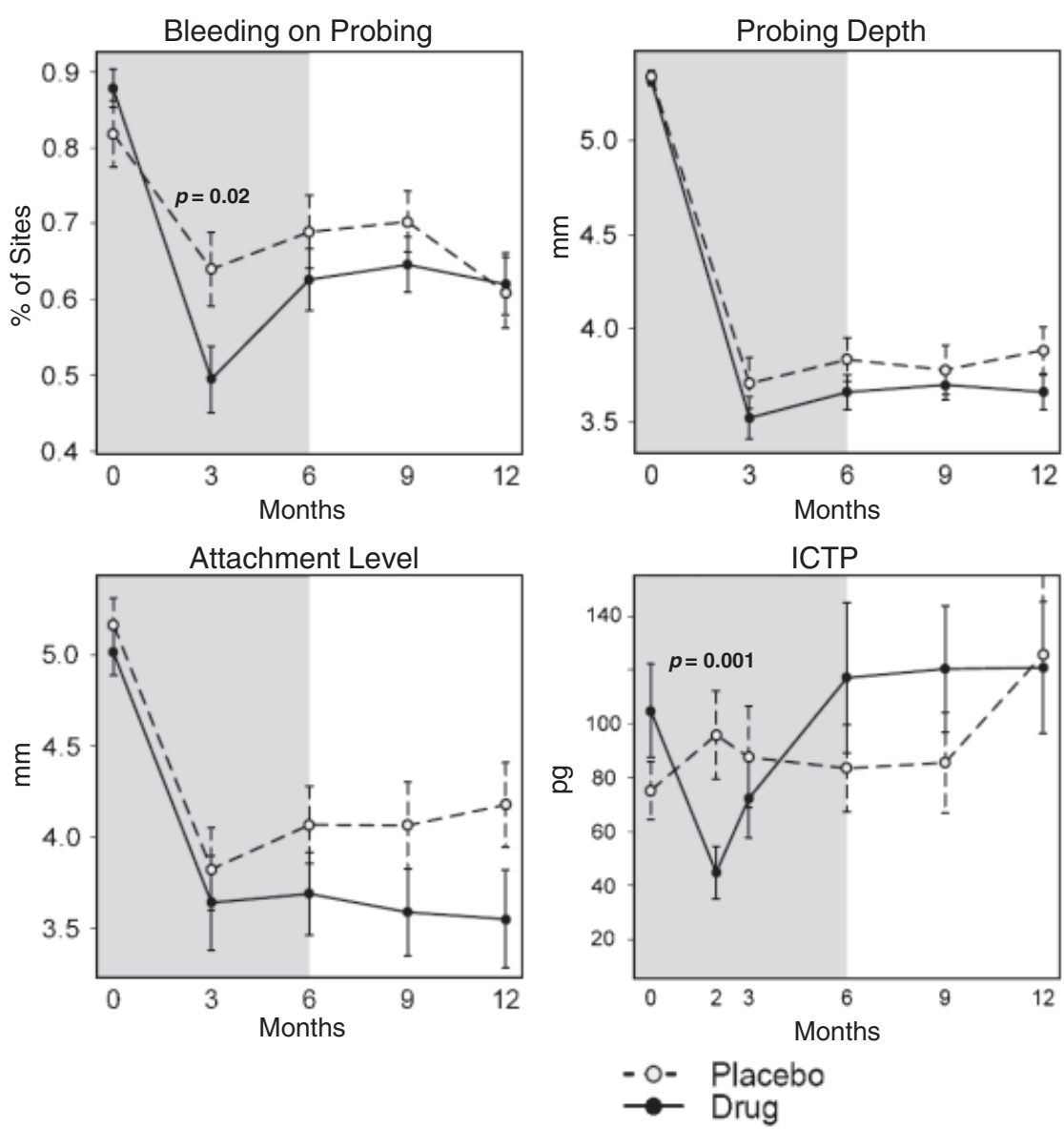

Fig. 3. Effect of subantimicrobial dose doxycycline (SDD) or placebo in combination with surgery on clinical parameters and collagen telopeptides (ICTP) levels for initial pockets of $5-6 \mathrm{~mm}$. The shaded background depicts the period of drug or placebo administration. The bars represent per-patient standard errors. $p$ values indicate significant treatment group differences at a time point as determined by generalized estimating equations (GEE). $N=70$ subjects.

\section{Deep sites $(B L P D \geqslant 7 \mathrm{~mm})$}

Figure 4 shows the mean per patient averages in BOP, PD, CAL and ICTP levels for initially deep probing sites. Greater reductions in PD were noted in this PD stratum for both SDD and placebo groups. The drug therapy resulted in greater reductions in $\mathrm{PD}$ compared with controls at 3 months $(p=0.004)$. In addition, SDD-treated patients demonstrated a statistically significant increase in CAL compared with placebo during the drug administration ( 3 months, $p=0.02 ; 6$ months, $p=0.005)$.

\section{Adverse events}

The distributions of the adverse events were correspondingly distributed between the test and placebo groups. Overall, 58 patients (28 drug and 28 placebo) reported total of 93 adverse events during the course of the study and that could be classified according to oral and systemic criteria (Table 3). Overall, adverse events affecting the oral soft and hard tissues included postsurgical pain and/or erythema $(38.7 \%$ of events), tooth sensitivity $(8.6 \%$ of events) and periodontal abscess $(6.4 \%$ of events). Excluding pulpitis, tooth fracture and soft tissue lesions, the other events appeared to be due to the anticipated responses to surgery and was not attributable to the study medication. Two patients from the test group developed soft tissue oral lesions. One patient developed a soft tissue lesion at 3 months of the trial and it was diagnosed at 12 months as lichen planus. She received topical doses of fluocinonide to manage the area. The other patient developed oral ulcers 1 week after the surgery. The patient did not report other 
episodes during the rest of the trial, although had a past history of such lesions. The systemic adverse events during the study included headaches (6.4\% of events) and common cold (6.4\% of events). Myocardial infarction occurred in a patient who had not received the medication. This event occurred soon before the BL appointment and therefore, the patient was exited from the study.

\section{Discussion}

This multi-center investigation sought to evaluate the potential additive effect of SDD to modulate wound repair in conjunction with periodontal surgery. The goal was to explore the clinical and biochemical impact of this drug on the modulation of wound healing post-surgically. Following mechanical therapy in the form of scaling and root planing, patients then received access flap surgery and were randomized to SDD or placebo groups for 6 months of drug therapy, followed by a 6-month "washout'". The patients exhibited moderate to severe horizontal bone loss, not indicated for conventional regenerative therapy. Therefore, patients included in this trial exhibited bone loss patterns with limited treatment options. When SDD was administered in combination with surgery, greater wound repair was found in the form of PD reduction and increase in CAL compared with placebo (Figs 2-4). SDD therapy may promote more rapid wound maturation by inhibiting collagen disruption and promoting collagen neogenesis. Therefore, differences in timing, quality and quantity of wound consolidation in the periodontium might have promoted an additive therapeutic effect in the SDD patients. Further statistical analysis also revealed that there were limited centre effects (data not shown). In fact, the significant differences indicated in the plots remained significant (with nearly identical $p$-values) after adjusting for clinical site confounders.

In this study, the inhibition of MMP via SDD resulted in a decrease in the bone resorption biomarker, ICTP, levels at 2 months after the surgical therapy while placebo demonstrated increases in ICTP levels over the same observation period. ICTP reflects the amount of organic matrix being disrupted in alveolar bone (specifically collagen type I) following osteoclastic bone resorption.
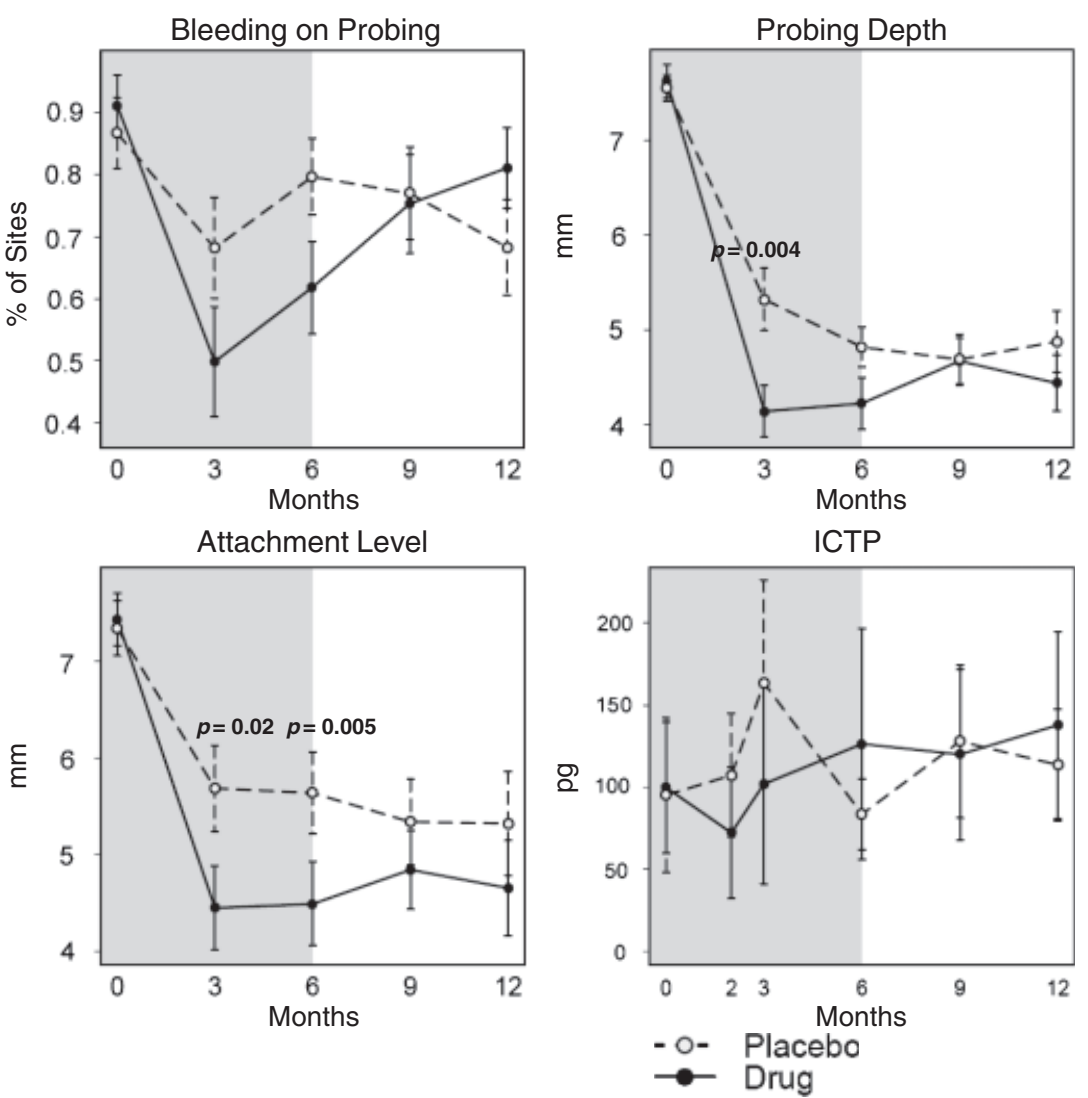

Fig. 4. Effect of low dose doxycycline (SDD) or placebo in combination with surgery on clinical parameters and collagen telopeptides (ICTP) for initial pocket of $\geqslant 7 \mathrm{~mm}$. The shaded background depicts the time of drug or placebo administration. The bars represent per-patient standard errors. $p$ values indicate significant treatment group differences at a time point as determined by generalized estimating equations (GEE). $N=70$ subjects.

ICTP has been utilized as a bone resorption marker in several systemic disease states including osteoarthritis (Berger et al. 2005), rheumathoid arthritis (Chopin et al. 2008), osteochondrosis (Vervuert et al. 2007), primary tumours and metastasis (Kong et al. 2007) and many other skeletal conditions. It has been shown by histological human data that osteoclastic activity peaks immediately after the bone is exposed by surgical operations (Wilderman et al. 1970). Therefore, release of excessive osteoclastic collagenases (or MMP-13) may have increased the disruption of collagen type I in bone and consequently increased ICTP release in the placebo. SDD-treated subjects displayed lower levels of wound fluid ICTP after surgery compared with BL, suggesting a "bonesparing effect', of the drug. In fact, some studies have demonstrated that chemically modified TCNs not only inhibit bone resorption through their ability to inhibit MMPs but also by inducing apoptosis of osteoclasts, by inhibiting osteoclastic resorption and by inhibiting osteoclastogenesis (Holmes et al. 2004). Hence, our findings have demonstrated that utilization of an MMPi may have a direct impact on post-surgical bone turnover. Although these findings are limited to this subset of patients from our trial, these results should encourage further exploration of the impact of host modulatory agents on bone maintenance.

The MMPs are a family of 23 endopeptidases in humans (Clark et al. 2008) that were originally described as cleaving extracellular matrix macromolecules, but it is now clear that they have much wider functionality (Clark et al. 2008). Control over MMP and/or tissue inhibitor of metalloproteinases activity in vivo occurs at different levels and involves factors such as regulation of gene expression, activation of zymogens and inhibition of active enzymes by specific inhibitors. Nearly 60 different MMPis have been pursued as clinical candidates to date (Dorman et al. 2007). 
Table 3. Percentages and distribution of adverse events during the course of the study

\begin{tabular}{lccc}
\hline Adverse event characteristics & Distribution of events $N(\%)$ & Drug $N(\%)$ & Placebo $N(\%)$ \\
\hline Dental related & & & \\
Post-surgical pain and/or erythema & $36(51.4)$ & $20(57.1)$ & $16(45.7)$ \\
Tooth sensitivity & $8(11.4)$ & $4(11.4)$ & $4(11.4)$ \\
Periodontal abscess & $6(8.5)$ & $3(8.5)$ & $3(8.5)$ \\
Tooth fracture & $3(4.2)$ & $1(2.8)$ & $2(5.7)$ \\
Pulpitis & $2(2.8)$ & $1(2.8)$ & $1(2.8)$ \\
Soft tissue lesion & $2(2.8)$ & $2(5.7)$ & $0(0)$ \\
Infection & $1(1.4)$ & $0(0)$ & $1(2.8)$ \\
Gingival swelling & $1(1.4)$ & $0(0)$ & $1(2.8)$ \\
Systemic Related & & & \\
Headache & $6(8.5)$ & $4(11.4)$ & $2(5.7)$ \\
Common Cold & $6(8.5)$ & $3(8.5)$ & $3(8.5)$ \\
Stomach pain & $3(4.2)$ & $1(2.8)$ & $2(5.7)$ \\
Depression/anxiety & $3(4.2)$ & $1(2.8)$ & $2(5.7)$ \\
Physical Injury & $2(2.8)$ & $1(2.8)$ & $1(2.8)$ \\
Ear pain & $2(2.8)$ & $1(2.8)$ & $1(2.8)$ \\
Lower back pain & $2(2.8)$ & $1(2.8)$ & $1(2.8)$ \\
Lethargy/drowsiness & $2(2.8)$ & $0(0)$ & $2(5.7)$ \\
Arthritis & $1(1.4)$ & $1(2.8)$ & $0(0)$ \\
Ibuprofen exposure & $1(1.4)$ & $0(0)$ & $1(2.8)$ \\
Sleep problems & $1(1.4)$ & $0(0)$ & $1(2.8)$ \\
Facial rash & $1(1.4)$ & $0(0)$ & $1(2.8)$ \\
Sinus allergy & $1(1.4)$ & $0(0)$ & $1(2.8)$ \\
High cholesterol & $1(1.4)$ & $0(0)$ & $1(2.8)$ \\
Vascular surgery at both legs & $1(1.4)$ & $0(0)$ & $1(2.8)$ \\
Shoulder pain & $1(1.4)$ & $0(0)$ & $1(2.8)$ \\
Myocardial infarction & $1(1.4)$ & & $1(1.4)^{*}$ \\
\hline & & &
\end{tabular}

$N$, number of patient with adverse events.

*The patient was excluded before baseline visit, therefore no group was assigned.

Targeted indications included rheumatoid arthritis, cardiovascular diseases, periodontal disease and many others (Giannobile 2008). MMPs have been considered prognostic factors in various types of cancer as well as promising targets for cancer therapy (Mannello 2006). Although numerous drug therapies to inhibit MMPs have been explored, only one agent SDD is currently approved for clinical use (Corbitt et al. 2007). This multi-centre randomized-clinical trial has demonstrated positive effect of an MMPi, SDD on post-surgical wound healing in patients with severe chronic periodontitis, at soft and hard tissue level. These results should encourage future basic and translational research in order to find even more effective MMPis. The next generations of MMPi should have specificity, gained from an understanding not only of the catalytic domain structures but the role of extracatalytic motifs in substrate binding (Murphy \& Nagase 2008). In fact, innovative approaches for the design of selective MMP inhibitors are currently being performed including the integration of medicinal chemistry structure-based properties and design features (Dorman et al. 2007). This approach, which includes privileged structures, molecular frameworks, bioisosteric and bioanalogous/ isofunctional modifications may lead to more highly selective MMPi in the future.

\section{Conclusions}

Six-month administration of MMPi, SDD, displayed an enhanced periodontal post-surgical wound healing compared with placebo controls. Early, transient reductions in the bone resorption marker ICTP were also found in patients while on the drug, suggesting the potential of SDD to act as a bonesparing agent.

\section{Acknowledgements}

The authors thank Dr. Christopher Powala for his assistance in the organization of the trial.

\section{References}

Berger, C. E., Kroner, A., Stiegler, H., Leitha, T. \& Engel, A. (2005) Elevated levels of serum type I collagen C-telopeptide in patients with rapidly destructive osteoarthritis of the hip. International Orthopaedics 29, 1-5.
Caton, J. G., Ciancio, S. G., Blieden, T. M., Bradshaw, M., Crout, R. J. et al. (2000) Treatment with subantimicrobial dose doxycycline improves the efficacy of scaling and root planing in patients with adult periodontitis. Journal of Periodontology $\mathbf{7 1}$, 521-532.

Caton, J. G., Ciancio, S. G., Blieden, T. M., Bradshaw, M., Crout, R. J. et al. (2001) Subantimicrobial dose doxycycline as an adjunct to scaling and root planing: posttreatment effects. Journal of Clinical Periodontology 28, 782-789.

Chopin, F., Garnero, P., le Henanff, A., Debiais, F., Daragon, A. et al. (2008) Long-term effects of infliximab on bone and cartilage turnover markers in patients with rheumatoid arthritis. Annals of the Rheumatic Diseases 67, 353-357.

Clark, I. M., Swingler, T. E., Sampieri, C. L. \& Edwards, D. R. (2008) The regulation of matrix metalloproteinases and their inhibitors. The International Journal of Biochemistry and Cell Biology 40, 1362-1378.

Corbitt, C. A., Lin, J. \& Lindsey, M. L. (2007) Mechanisms to inhibit matrix metalloproteinase activity: where are we in the development of clinically relevant inhibitors? Recent Patents on Cardiovascular Drug Discovery 2, 135-145.

Dorman, G., Kocsis-Szommer, K., Spadoni, C. \& Ferdinandy, P. (2007) MMP inhibitors in cardiac diseases: an update. Recent Patents on Cardiovascular Drug Discovery 2, 186194.

Gapski, R. G., Barr, J. L., Sarment, D. P., Layher, M. G., Socransky, S. S. \& Giannobile, W. V. (2004) Systemic MMP inhibition promotes periodontal wound repair. A proof of concept trial. Jounal of Periodontology 75, 441-452.

Giannobile, W. V. (2008) Host-response therapeutics for periodontal diseases. Journal of Periodontology 79 (Suppl.), 1592-1600.

Giannobile, W. V., Lynch, S. E., Denmark, R. G., Paquette, D. W., Fiorellini, J. P. et al. (1995) Crevicular fluid osteocalcin and pyridinoline cross-linked carboxyterminal telopeptide of type I collagen (ICTP) as markers of rapid bone turnover in periodontitis. A pilot study in beagle dogs. Journal of Clinical Periodontology 22, 903-910.

Gill, S. E. \& Parks, W. C. (2008) Metalloproteinases and their inhibitors: regulators of wound healing. The International Journal of Biochemistry and Cell Biology 40, 13341347.

Golub, L. M., Ciancio, S., Ramamamurthy, N. S., Leung, M. \& McNamara, T. F. (1990) Lowdose doxycycline therapy: effect on gingival and crevicular fluid collagenase activity in humans. Journal of Periodontal Research $\mathbf{2 5}$, 321-330.

Golub, L. M., Lee, H. M., Greenwald, R. A., Ryan, M. E., Sorsa, T. et al. (1997) A matrix metalloproteinase inhibitor reduces bonetype collagen degradation fragments and specific collagenases in gingival crevicular fluid during adult periodontitis. Inflammation Research 46, 310-319. 
Golub, L. M., Lee, H. M., Lehrer, G., Nemiroff, A., McNamara, T. F. et al. (1983) Minocycline reduces gingival collagenolytic activity during diabetes. Preliminary observations and a proposed new mechanism of action. Journal of Periodontal Research 18 , 516-526.

Golub, L. M., Lee, H. M., Ryan, M. E., Giannobile, W. V., Payne, J. et al. (1998) Tetracyclines inhibit connective tissue breakdown by multiple non-antimicrobial mechanisms. Advances in Dental Research 12, 12-26.

Golub, L. M., McNamara, T. F., D’Angelo, G., Greenwald, R. A. \& Ramamurthy, N. S. (1987) A non-antibacterial chemically-modified tetracycline inhibits mammalian collagenase activity. Journal of Dental Research 66, 1310-1314.

Golub, L. M., McNamara, T. F., Ryan, M. E., Kohut, B., Blieden, T. et al. (2001) Adjunctive treatment with subantimicrobial doses of doxycycline: effects on gingival fluid collagenase activity and attachment loss in adult periodontitis. Journal of Clinical Periodontology 28, 146-156.

Golub, L. M., Siegel, K., Ramamurthy, N. S. \& Mandel, I. D. (1976) Some characteristics of collagenase activity in gingival crevicular fluid and its relationship to gingival diseases in humans. Journal of Dental Research 55, 1049-1057.

Goodson, J. M. (2004) Confusion between definitive and exploratory clinical therapy trials: when to believe and when to question. Journal of Periodontology 75, 493-494.

Gopalsami, C., Yotis, W., Corrigan, K., Schade, S., Keene, J. et al. (1993) Effect of outer membrane of Treponema denticola on bone resorption. Oral Microbiology and Immunology 8, 121-124.

Gu, Q., Wang, D., Gao, Y., Zhou, J., Peng, R. et al. (2002) Expression of MMP1 in surgical and radiation-impaired wound healing and its effects on the healing process. Journal of Environmental Pathology, Toxicology and Oncology: Official Organ of the International Society for Environmental Toxicology and Cancer 21, 71-78.

Holmes, S. G., Still, K., Buttle, D. J., Bishop, N. J. \& Grabowski, P. S. (2004) Chemically modified tetracyclines act through multiple mechanisms directly on osteoclast precursors. Bone 35, 471-478.

Kong, Q. Q., Sun, T. W., Dou, Q. Y., Li, F., Tang, Q. et al. (2007) Beta-CTX and ICTP act as indicators of skeletal metastasis status

\section{Clinical Relevance}

Scientific rationale for the study: The determination that periodontal tissue destruction is primarily due to the host response has created areas of research directed at altering an individual's reaction to the bacterial challenge. Hence, new methods of host modulatory therapy for periodontal disease are being focused in male patients with non-small cell lung cancer. The International Journal of Biological Markers 22, 214-220.

Kornman, K. S. (2008) Mapping the pathogenesis of periodontitis: a new look. Journal of Periodontology $\mathbf{7 9}$ (Suppl.), $1560-1568$.

Lopez-Otin, C. \& Matrisian, L. M. (2007) Emerging roles of proteases in tumour suppression. Nature Reviews. Cancer 7, 800-808.

Mannello, F. (2006) Natural bio-drugs as matrix metalloproteinase inhibitors: new perspectives on the horizon? Recent Patents on Anti-Cancer Drug Discovery 1, 91-103.

Moher, D., Schulz, K. F. \& Altman, D. (2001) The CONSORT statement: revised recommendations for improving the quality of reports of parallel-group randomized trials. JAMA 285, 1987-1991.

Murphy, G. \& Nagase, H. (2008) Reappraising metalloproteinases in rheumatoid arthritis and osteoarthritis: destruction or repair? Nature Clinical Practice. Rheumatology 4, 128135.

Neely, A. N., Clendening, C. E., Gardner, J. \& Greenhalgh, D. G. (2000) Gelatinase activities in wounds of healing-impaired mice versus wounds of non-healing-impaired mice. The Journal of Burn Care and Rehabilitation 21, 395-402.

Novak, M. J., Johns, L. P., Miller, R. C. \& Bradshaw, M. H. (2002) Adjunctive benefits of subantimicrobial dose doxycycline in the management of severe, generalized, chronic periodontitis. Journal of Periodontology 73, 762-769.

Preshaw, P. M., Hefti, A. F., Novak, M. J., Michalowicz, B. S., Pihlstrom, B. L. et al. (2004) Subantimicrobial dose doxycycline enhances the efficacy of scaling and root planing in chronic periodontitis: a multicenter trial. Journal of Periodontology 75, 10681076.

Preshaw, P. M., Novak, M. J., Mellonig, J., Magnusson, I., Polson, A. et al. (2008) Modified-release subantimicrobial dose doxycycline enhances scaling and root planing in subjects with periodontal disease. Journal of Periodontology 79, 440-452.

Pussinen, P. J., Paju, S., Mantyla, P. \& Sorsa, T. (2007) Serum microbial- and host-derived markers of periodontal diseases: a review. Current Medicinal Chemistry 14, 2402-2412.

Shi, Y. B., Fu, L., Hasebe, T. \& Ishizuya-Oka, A. (2007) Regulation of extracellular matrix remodeling and cell fate determination by matrix metalloproteinase stromelysin-3 during thyroid hormone-dependent post-embryonic development. Pharmacology and Therapeutics 116, 391-400.

Slots, J. \& Genco, R. J. (1984) Black-pigmented bacteroides species, capnocytophaga species, and actinobacillus actinomycetemcomitans in human periodontal disease: virulence factors in colonization, survival, and tissue destruction. Journal of Dental Research 63, 412 421.

Socransky, S. S. \& Haffajee, A. D. (1991) Microbial mechanisms in the pathogenesis of destructive periodontal diseases: a critical assessment. Journal of Periodontal Research 26, 195-212.

Sorsa, T., Uitto, V. J., Suomalainen, K., Vauhkonen, M. \& Lindy, S. (1988) Comparison of interstitial collagenases from human gingiva, sulcular fluid and polymorphonuclear leukocytes. Journal of Periodontal Research 23, 386-393.

Tarlton, J. F., Vickery, C. J., Leaper, D. J. \& Bailey, A. J. (1997) Postsurgical wound progression monitored by temporal changes in the expression of matrix metalloproteinase-9. The British Journal of Dermatology 137, 506-516.

Varghese, S. (2006) Matrix metalloproteinases and their inhibitors in bone: an overview of regulation and functions. Frontiers in Bioscience 11, 2949-2966.

Vervuert, I., Winkelsett, S., Christmann, L., Bruns, E., Hoppen, H. O. et al. (2007) Evaluation of the influences of exercise, birth date, and osteochondrosis on plasma bone marker concentrations in Hanoverian Warmblood foals. American Journal of Veterinary Research 68, 1319-1323.

Wilderman, M. N., Pennel, B. M., King, K. \& Barron, J. M. (1970) Histogenesis of repair following osseous surgery. Journal of Periodontology 41, 551-565.

Address:

William V. Giannobile

Michigan Center for Oral Health Research

University of Michigan School of Dentistry

24 Frank Lloyd Wright Dr.

Lobby M Box 422

Ann Arbor MI 48106

USA

E-mail:wgiannob@umich.edu beyond the traditional control of tooth-associated microbial biofilms. Principal findings: This manuscript demonstrates that the utilization of a 6-month dosing of a MMP inhibitor, SDD in combination with access flap surgery enhances periodontal postsurgical wound healing compared with placebo. Furthermore, transient reductions in the bone resorption marker were also found in patients while on the drug, suggesting the potential of SDD to act as a bonesparing agent.

Practical implications: These results support and encourage further exploration of the impact of host modulatory agents for periodontal repair and bone maintenance. 